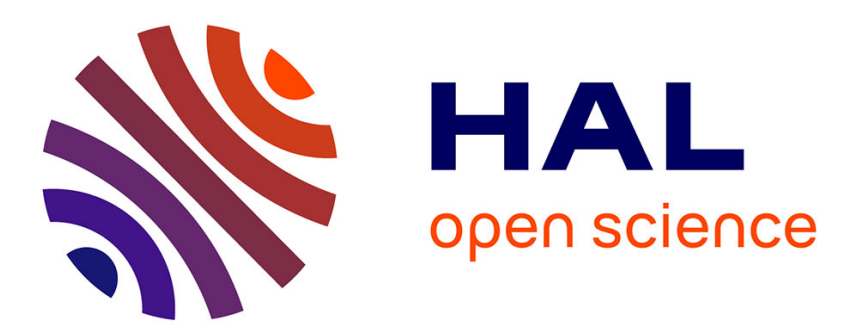

\title{
Adaptative Force Feedback Control for 3D Compensation of Physiological Motion in Beating Heart Surgery
}

\author{
Zeineb Zarrouk, Ahmed Chemori, Philippe Poignet
}

\section{To cite this version:}

Zeineb Zarrouk, Ahmed Chemori, Philippe Poignet. Adaptative Force Feedback Control for 3D Compensation of Physiological Motion in Beating Heart Surgery. IROS: Intelligent Robots and Systems, Oct 2010, Taipei, Taiwan. pp.1856-1861, 10.1109/IROS.2010.5650684 . lirmm-00545458

\section{HAL Id: lirmm-00545458 \\ https://hal-lirmm.ccsd.cnrs.fr/lirmm-00545458}

Submitted on 10 Sep 2019

HAL is a multi-disciplinary open access archive for the deposit and dissemination of scientific research documents, whether they are published or not. The documents may come from teaching and research institutions in France or abroad, or from public or private research centers.
L'archive ouverte pluridisciplinaire HAL, est destinée au dépôt et à la diffusion de documents scientifiques de niveau recherche, publiés ou non, émanant des établissements d'enseignement et de recherche français ou étrangers, des laboratoires publics ou privés. 


\title{
Adaptive Force Feedback Control for 3D Compensation of Physiological Motion in Beating Heart Surgery
}

\author{
Zeineb Zarrouk, Ahmed Chemori and Philippe Poignet
}

\begin{abstract}
In this paper the problem of 3D physiological motion compensation in beating heart surgery is resolved by an adaptive control architecture based on Model Reference Adaptive Control (MRAC). The proposed control architecture uses the measures of the contact efforts applied by the surgical tool on the heart to assure force feedback. No apriori information about motion characteristics is necessary. It includes a nonlinear feedback linearizing the robot dynamics and a velocity loop. Simulation results are presented to show the effectiveness of the proposed control architecture for 3D compensation of physiological motions in beating heart surgery. Furthermore, its robustness toward uncertainties on dynamic parameters and environment stiffness is shown.
\end{abstract}

\section{INTRODUCTION AND RELATED WORK}

In cardiac surgery, physiological motions induced by the heartbeat and respiration can make it very problematic for the surgeon to perform skillful and precise operations on the heart. These motions represent a source of perturbations that make surgical tasks very difficult to perform. It is proved that manual tracking of heart motion cannot be achieved without phase and amplitude errors [1]. For many years, cardiac surgery has been performed using an external machine that insures blood circulation and body's oxygenation. This technique allows the surgeon to operate on a stopped heart and perform precise gestures. However, the use of the heart-lung machine implies more risks and a longer recovery time [2].

An alternative technique to cancel beating heart motion is the use of a passive mechanical stabilizer. This device stabilizes a small region on the heart surface by suction or pressure. However, the stabilizer may damage the myocardium tissue and experiments performed on pigs show a residual cardiac motion that is still important to achieve heartbeat surgery [3].

To avoid the use of the heart-lung machine and overcome the problems related to the physiological motions, robotized systems using vision or force control to compensate them have been developed. In this context, several control architectures have been proposed to deal with motion compensation in minimally invasive beating heart surgery.

Based on a vision sensor, Nakamura et al. [4] developed the first system for estimating the heart motion using high-speed cameras by tracking markers fixed on the heart surface.

In [5], Ginhoux et al. proposed a vision based approach for motion canceling using model predictive control to get higher precision tracking. Once again, a camera is

Zeineb Zarrouk, Ahmed Chemori and Philippe Poignet are with LIRMM, Univ. Montpellier 2 - CNRS, 161 rue Ada, 34392 Montpellier, France chemorielirmm. fr employed to track artificial markers placed on the heart surface. However, the use of additional markers is not desirable in real surgical procedures due to the space limitation and to the problems related to their fixation. To avoid such problems, Ortmaier et al. [6] have used the natural textures on the heart surface for estimating the heart motion using vision. In this approach, salient features on the heart surface were tracked using a motion model. In [7], Bebek et al. demonstrated that the tracking motion can be improved by the use of electrocardiogram (ECG) signal in the motion estimation. Their approach is based on the fusion of the heart motion measured by mechanical motion sensors and the ECG signal. This information is used to predict the feedforward reference signal. Both control approaches developed in [5] and [7] used algorithms predicting the future heart motion based on recorded heart cycles. However during a contact task, the forces applied on the heart introduce disturbances modifying the natural motion. In this case, the predictive algorithms may fail to accurately predict and compensate the future heart motion. Besides the problem of the heartbeat in minimally invasive cardiac surgery, an important obstacle encountered by surgeons is the significant degradation of haptic feedback about the surgical instrument interaction with tissues. Indeed, surgeons can only estimate contact forces through the tissue deformation. Therefore, focusing on motion compensation using force feedback, Cagneau et al. [8] proposed an approach based on a PI scheme coupled with an Iterative Learning Control (ILC). The proposed algorithm assumes that the heart motion is periodic. This hypothesis may be too restrictive and the experiments performed on an animated contact show large tracking errors. In [9], a novel technique is proposed to compensate physiological motions using force control. This approach is based on two independent Active OBservers (AOB) designed for force control and motion compensation. The first AOB has its estimation strategy tuned for haptic telemanipulation, providing control actions through estimated states to achieve a desired closed loop dynamics. The second AOB performs control actions referred to the system input. The obtained simulation of this control approach shows good compensation of sinusoidal and non-sinusoidal physiological motions.

In [10], Dominici et al. proposed a predictive force control for compensating physiological motions based on a process model to predict future plant behavior using past and current forces applied values. Simulation results show the efficiency of the proposed control approach to compensate heart motion along $Z$ axis. 
This present paper deals with a technique to compensate physiological motions in minimally invasive beating heart surgery based on an adaptive force feedback scheme.

The paper is organized as follows : Section II describes the D2M2 robot manipulator whose dynamic model is used for simulations. Section III describes the proposed control architecture used in the compensation problem. Simulation results are presented and commented in Section IV. Finally, Section V deals with some concluding remarks.

\section{THE D2M2 ROBOT MANIPULATOR}

The D2M2 (cf. Fig. 1) is a robot manipulator designed for surgery experiments. It has 5 degrees of freedom with direct drive actuators providing high-bandwidth motion tracking and low friction. These properties allow the robot endeffector to track the heart motion characterized by high frequencies and may ensure motion compensation. An ATI

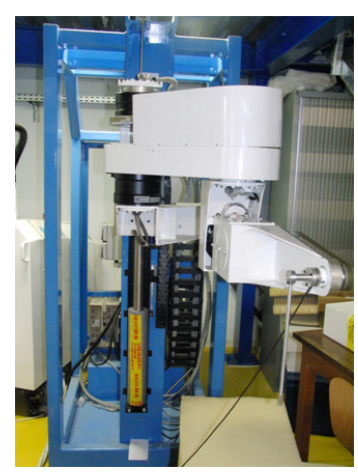

Fig. 1. The D2M2 robot

Mini 40 force sensor is fixed at the extremity of the endeffector to measure in real time the forces applied by the robot on the environment.

The dynamic model [11] of the D2M2 robot in contact with its environment is given by :

$$
\Gamma=M(q) \ddot{q}+V(q, \dot{q})+G(q)+J^{T}(q) F_{e}
$$

where

- $\Gamma$ : the vector of the generalized torques

- $\mathrm{M}$ : the inertia matrix

- $\mathrm{V}$ : the Coriolis and Centrifugal vector

- $\mathrm{G}$ : the gravity vector

- $\mathrm{J}$ : the Jacobian matrix

- $F_{e}$ : the measured contact force at the end-effector

- $q, \dot{q}$ and $\ddot{q}$ : the vectors respectively of articular positions, velocities and accelerations.

This model can be rewritten as follows :

$$
M_{x}(q) \ddot{X}_{s}+V_{x}(q, \dot{q})+G_{x}(q)=F_{s c}-F_{e}
$$

with:

$$
\begin{gathered}
M_{x}(q)=J^{-T}(q) M(q) J^{-1}(q) \\
V_{x}(q)=J^{-T}(q) V(q, \dot{q})-M_{x} \dot{J}(q, \dot{q}) \dot{q}
\end{gathered}
$$

$$
G_{x}(q)=J^{-T}(q) G(q)
$$

$M_{x}, V_{x}$ and $G_{x}$ are respectively the inertial matrix, the Coriolis and centrifugal force vector and the gravity term written in Cartesian space. $F_{s c}$ denotes the commanded force and $\dot{J}=\frac{d J}{d t}$ is the first derivative of the Jacobian matrix.

Let us now consider the control input $F_{S c}$ as :

$$
F_{s c}=M_{x}(q) f^{*}+V_{x}(q, \dot{q})+G_{x}(q)+F_{e}
$$

This leads to the following fully linearized and decoupled second order system :

$$
\ddot{X}_{s}=f^{*}
$$

where $f^{*}$ is an acceleration, being an input parameter.

In this work, the singularity of the Jacobian matrix is not considered. In fact, the low amplitude of the heart motion signal may not put the robot in a singular configuration. Equation (4) represents the dynamics of a unitary mass for each Cartesian dimension described by Fig. 2 .

Introducing the gain $K_{2}$ of the velocity loop (cf. Fig. 3), the

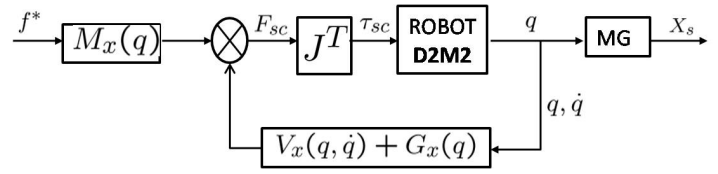

Fig. 2. Decoupled and linearized system

equivalent linear transfer function is given by :

$$
G(p)=\frac{F_{e}}{u}=\frac{1}{p\left(p+K_{2}\right)}
$$

where $p$ represents Laplace operator and $F_{e}$ is the Cartesian force applied by the robot and $u$ is the control input.

\section{PROPOSED ADAPTIVE FORCE FEEDBACK CONTROL ARCHITECTURE}

\section{A. Global control architecture}

The global control scheme, shown in Fig. 3, is composed of five parts, namely:

- The system plant linearized in Cartesian space

- The contact environment

- The adaptive controller

- The reference model which characterizes the closedloop desired performance

- A linear pre-filter $H_{m}$ that determines the reference signal. It is given by :

$$
H_{m}(p)=\frac{\omega^{2}}{p^{2}+2 \xi \omega p+\omega^{2}}
$$

In the following section, the different blocks of the global control architecture are detailed.

\section{B. Exact feedback linearization of the robot dynamics}

To ensure the heart compensation, the proposed control architecture is based on a linear adaptive controller. The use of a linear controller implies full exact input-state linearization of the robot dynamics. The linearized model is presented and described in section II. 


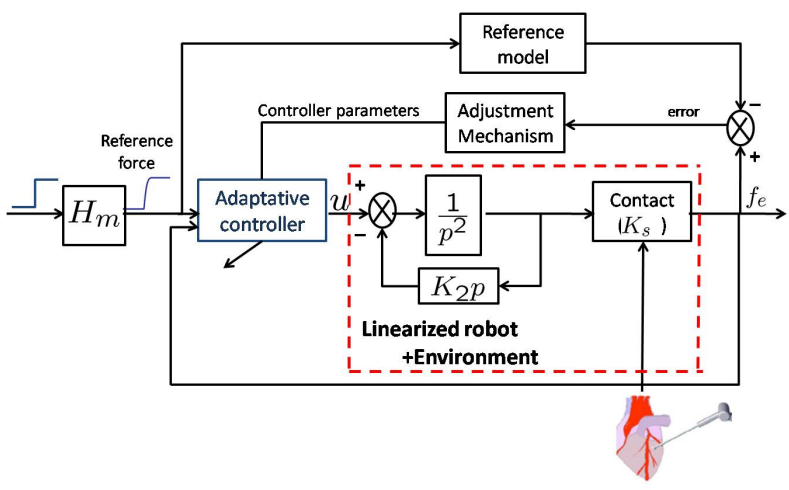

Fig. 3. Global control scheme with linear adaptive force controller

\section{Environment}

The block "Contact $\left(K_{S}\right)$ " presented in Fig. 3 is the environment with which the robot's end-effector interacts. In our case, it represents the heart surface which is in perpetual motion. The contact between the end-effector and the heart surface is modeled as a spring with a stiffness $K_{s}$. According to this assumption, the force $F_{e}$ applied by the robot's endeffector on its environment is giving by:

$$
F_{e}=K_{s} X
$$

where $X$ assigns the penetration of the end-effector in the heart tissue.

\section{Model Reference Adaptive Force Control}

The Model Reference Adaptive Controller (MRAC) is one of the most important adaptive controllers [12]. It can be seen as an adaptive servo system in which the desired performance is specified through a reference model. The goal of the adaptive controller is to adjust the parameters of the controller to deal with eventual variations in the dynamics of the controlled system and/or uncertainties. The adjustment mechanism is defined by minimizing a determined cost function to keep the output of the process as close as possible to that of the reference model. The proposed controller MRAC is based on output feedback [13]. It is designed for a SISO process with one input and one output. To ensure 3D motion compensation, three MRAC Controllers are used (one controller for each dimension). The proposed MRAC controllers are based on the MIT rule in the adjustment mechanism. This rule is defined by the following expression

$$
\frac{d \theta}{d t}=\gamma \phi \varepsilon
$$

where

- $\theta$ is a vector of the controller's parameters,

- $\gamma$ is a parameter that characterizes the adaptation rate,

- $\phi$ is a vector of filtered output, input and command signal,

- $\varepsilon$ is the augmented error.

The derivation of the MRAC in RST form is described as follows:
- The structure of the controller (cf. Fig. 4) [14] is defined by the following equation :

$$
R u(t)=-S y(t)+T u_{c}(t)
$$

where $u$ and $y$ are respectively the input and the output of the controlled system, and $u_{c}$ is the reference signal. According to the control architecture of Fig. 3, the reference signal $u_{c}$ corresponds to the force which the robot's end-effector must apply on its environment. This structure allows the process to get a perfect output tracking. The polynomials $R, S$, and $T$, specified by equations (7), (8), and (9), are resolved by the so-called Diophantine equation.

$$
\begin{gathered}
R(p)=p+r_{1} \\
S(p)=s_{0} p+s_{1} \\
T(p)=t_{0} p+t_{1}
\end{gathered}
$$

The vector $\theta$ of adjustment is then given by :

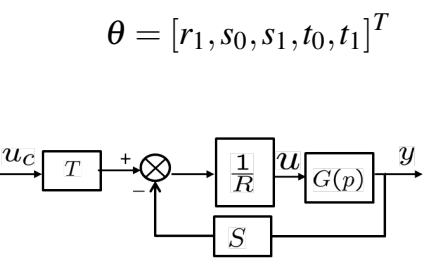

Fig. 4. RST controller

At each instant, the parameters of the polynomials $R, S$ and $T$ are updated according to the change in the system parameters.

- Derive an error model $\varepsilon$ by adding a correction term to the error between the output of the process and that of the model.

- The parameters adjustment law is defined by equation (6).

The MRAC controller aims to minimize the error between the outputs of the process and the model. The goal of this approach is to keep the applied force equal to the desired one. This means that the output of the reference model must be as close as possible to the desired force. To satisfy this condition, the reference model is described by a second order function with a static gain close to 1 and a phase close to 0 .

$$
H_{m}(p)=\frac{\omega^{2}}{p^{2}+2 \xi \omega p+\omega^{2}}
$$

with $\omega=500$ and $\xi=1$.

\section{E. Closed-loop stability analysis using augmented error}

According to [13] (chapter 5, section 5.7), THEOREM 5.9 elaborates the stability of the closed-loop system controlled by the MRAC controller. Indeed, the transfer $G$ can be factorized as $G_{1} G_{2}$ such that $G_{1}$ is strictly positive real. Then the proposed adaptation law (6) gives an asymptotically stable closed-loop system, that is the error tends to zero as $t$ tends to $\infty$. 


\section{SIMULATION RESULTS AND ROBUSTNESS ANALYSIS}

This section focuses on the simulation results of the proposed control architecture described in section III. Simulations are performed using MATLAB R2007a software. The beating heart signals simulating the heart motion are those of [15]. They have been recorded on a pig beating heart using a vision based system. The obtained 3D motions data are displayed in Fig. 5. The force reference is the output

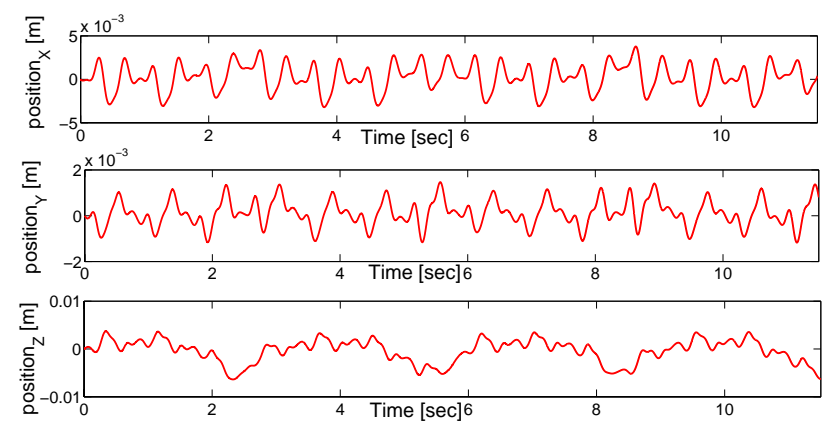

Fig. 5. Recorded physiological 3D motion data [15]

of the pre-filter $H_{m}$ described by equation (5) with $\omega=50$ and $\xi=1$. This pre-filter inhibits the sudden changes of forces that may generate important torques. Three simulation scenarios are performed, namely :

- motion compensation in nominal case,

- robustness toward parameters uncertainty,

- robustness toward stiffness uncertainty,

they are detailed in the following sections.

\section{A. Simulation 1: Nominal case}

The initial condition of this first simulation assumes that the robot's end-effector is in contact with the organ. The realtime motion data, which are previously presented, are used to simulate the 3D motion of the beating heart. According to the real-time experiments achieved in [16], the contact stiffness in our simulator is set to $800 \mathrm{~N} / \mathrm{m}$. Three MRAC controllers are used along $X, Y$, and $Z$ to ensure compensation in 3D. In this simulation scenario, the robot parameters are considered perfectly identified and the external disturbances are supposed to be zero. A variable force reference is applied along each axis. The three first graphs in Fig. 6, 7, and 8 display the evolution of the desired forces in solid line and the applied forces in dashed line along the axis $X, Y$, and $Z$ respectively. The maximal force error is about $3 \%$ corresponding to a position error of about $3.7 \times 10^{-3} \%$. Fig. 9 shows the heart position (dashed line) and the robot endeffector position (solid line). The difference between the two is due to the D2M2 end-effector penetration of the heart surface, in order to track the desired force. Fig. 10 represents the evolution of the torques generated by the motors of the robot, where it can be clearly seen that they remain within the admissible limits given by the manufacturer of the actuators (150 N.m). The peaks that appear in these curves correspond to the changes in the reference forces.

This first simulation is carried out without errors introduced. It shows the good performance of the proposed force feedback control architecture to ensure 3D heartbeat compensation. In the next simulations, different uncertainties are considered to show the effectiveness and the robustness of the proposed control architecture.

\section{B. Simulation 2: Robustness toward dynamic parameters uncertainty}

In this simulation scenario, uncertainties on parameters are considered. In view of the fact that the amplitude of the heart motion is more important along $\mathrm{Z}$ axis, we introduce $25 \%$ of uncertainties on parameters corresponding to the $\mathrm{Z}$ axis and $5 \%$ on the other parameters. The maximal force error recorded along $X$ axis is almost 5,3\% and $2 \%$ along $Y$ and $Z$ axis. This resuts can be explained by the number of parameters that are greater along $X$ axis. This simulation proves that the controller is able to adapt its parameters to conform to another system whose parameters are different from the nominal one. The results of the tracking heart motion are shown in Fig. 11.

\section{Simulation 3: Robustness toward environment stiffness uncertainty}

In this simulation, we consider the situation where the robot's end-effector interacts with tissues of a stiffness different from the nominal one. The environment stiffness is set to $1200 \mathrm{~N} / \mathrm{m}$. This means that the stiffness uncertainty introduced is $50 \%$ of the nominal value. The $3 \mathrm{D}$ compensation of heart motion is shown in Fig. 12. Requiring more energy to apply the desired forces, the torques represented in Fig. 13, are greater comparing to the nominal case, nevertheless, they remain within the admissible limits.

\section{Comparison with other control architectures}

The force control architectures developed in [10] and [9] are based on a force feedback and linear controllers. These architectures were performed in task space and tested in simulation. A comparison of the proposed approach with these approaches is summarized in table I :

\begin{tabular}{|l|l|l|}
\hline Our controller & $\begin{array}{l}\text { Predictive controller } \\
{[10]}\end{array}$ & AOB controller [9] \\
\hline \hline 3D compensation & 1D compensation & 3D compensation \\
\hline $\begin{array}{l}\text { Use of real heart mo- } \\
\text { tion data }\end{array}$ & $\begin{array}{l}\text { Use of real heart mo- } \\
\text { tion data }\end{array}$ & $\begin{array}{l}\text { use of a non lin- } \\
\text { ear function simulat- } \\
\text { ing the heart motion }\end{array}$ \\
\hline $\begin{array}{l}\text { Robustness test to- } \\
\text { ward stiffness uncer- } \\
\text { tainties (50\%) }\end{array}$ & $\begin{array}{l}\text { Robustness test to- } \\
\text { ward stiffness uncer- } \\
\text { tainties (20\%) }\end{array}$ & No test made \\
\hline $\begin{array}{l}\text { Robustness test to- } \\
\text { ward parameters un- } \\
\text { certainties }(25 \%)\end{array}$ & $\begin{array}{l}\text { Robustness test to- } \\
\text { ward parameters un- } \\
\text { certainties (20\%) }\end{array}$ & No test made \\
\hline
\end{tabular}

TABLE I

COMPARISON OF THE PROPOSED CONTROLLER WITH THE CONTROLLERS PROPOSED IN [10] AND [9] 


\section{CONCLUSION AND FUTURE WORK}

This paper deals with an adaptive based force control architecture for 3D physiological motion compensation in mini-invasive cardiac surgery. The proposed control approach takes into consideration the breathing and heartbeat motion without adding markers in the workspace to measure the motion. Instead, a force sensor, located at the extremity of robot's end-effector, is used to measure contact forces applied by the surgical tool on the heart surface.

Three simulation scenarios have been implemented on the D2M2 robot manipulator. The first one deals with the nominal case, whereas the two others deal with robustness test of the proposed control scheme toward uncertainties on dynamic parameters and environment stiffness.

The next step of our work will be focused on real-time implementation of the proposed control architecture on the D2M2 experimental setup. Furthermore, to improve the effectiveness of the proposed control architecture, future work can also include a study of the possibility of taking into account actuators' saturations in the design of the control approach.

\section{REFERENCES}

[1] V. Falk, "Manual control and tracking-a human factor analysis relevant for beating heart surgery," The Annals of Thoracic Surgery, vol. 74, no. 2, pp. 624-628, 2002.

[2] A. M. Okamura, L. N. Verner, C. E. Reiley, and M. Mahvash, "Haptics for robot-assisted minimally invasive surgery," in 13th International Symposium of Robotics Research (ISRR'07), (Hiroshima, Japan), pp. 26-29, 2007.

[3] M. Lemma, A. Mangini, A. Redaelli, and F. Acocella, "Do cardiac stabilizers really stabilize? experimental quantitative analysis of mechanical stabilization," Interactive CardioVascular and Thoracic Surgery, vol. 4, pp. 222-226, 2005.

[4] Y. Nakamura, K. Kishi, and H. Kawakami, "Heatbeat synchronization for robotic cardiac surgery," in IEEE International Conference on Robotics and Automation (ICRA'01), (Seoul, Korea), pp. 2014-2019, 2001.

[5] R. Ginhoux, J. Gangloff, M. De Mathelin, L. Soler, M. M. Arenas Sanchez, and J. Marescaux, "Active filtering of physiological motion in robotized surgery using predictive control," IEEE Transactions on Robotics, vol. 21, 2005.

[6] T. Ortmaier, M. Groger, D. H. Boehm, V. Falk, and G. Hirzinger, "Motion estimation in beating heart surgery.," in IEEE Transactions on Biomedical Engineering, vol. 52, pp. 1729-1740, 2005.

[7] O. Bebek and M. Cavusoglu, "Intelligent control algorithms for robotic-assisted beating heart surgery," IEEE Transactions on Robotics, vol. 23, no. 3, pp. 468-480, 2007.

[8] B. Cagneau, N. Zemiti, D. Bellot, and G. Morel, "Physiological motion compensation in robotized surgery using force feedback control," in IEEE International Conference on Robotics and Automation (ICRA'07), (Rome, Italy), pp. 1881-1886, 2007.

[9] R. Cortesao and P. Poignet, "Motion compensation for robotic-assisted surgery with force feedback," in IEEE International Conference on Robotics and Automation (ICRA'09), (Kobe, Japan), pp. 3464-3469, 2009.

[10] M. Dominici, M. Poignet, and E. Dombre, "Compensation of physiological motion using linear predictive force control," in IEEE International Conference on Intelligent Robots and Systems (IROS'08), (Nice, France), pp. 1173-1178, 2008.

[11] E. Dombre and W. Khalil, Modeling, Identification and Control of Robots. Penton, 2002.

[12] V. Bodal, J. Bohm, J. Fessl, and J. Machacek, Digital Self-tuning Controllers: Algorithms, Implementation and Applications. springer, 2005.

[13] K. Astrom, Adaptive Control. Springer-Verlag New York Inc, 2005.

[14] I. Landau and G. Zito, Digital Control Systems: Design, Identification and Implementation. Springer UK, 2006.
[15] M. Sauvée, P. Poignet, J. triboulet, E. Dombre, E. Malis, and R. Demaria, "3d heart motion estimation using endoscopic monocular vision system," in IFAC Symposium on Modeling and Control in Biomedical Systems (MCBMS'06), (Reims, France), pp. 141-146, 2006.

[16] W. Zarrad, P. Poignet, R. Cortesao, and O. Company, "Stability and transparency analysis of a haptic feedback controller for medical applications," in IEEE Conference on Decision and Control (CDC'07), (New Orleans, LA, USA), pp. 5767-5772, 2007.

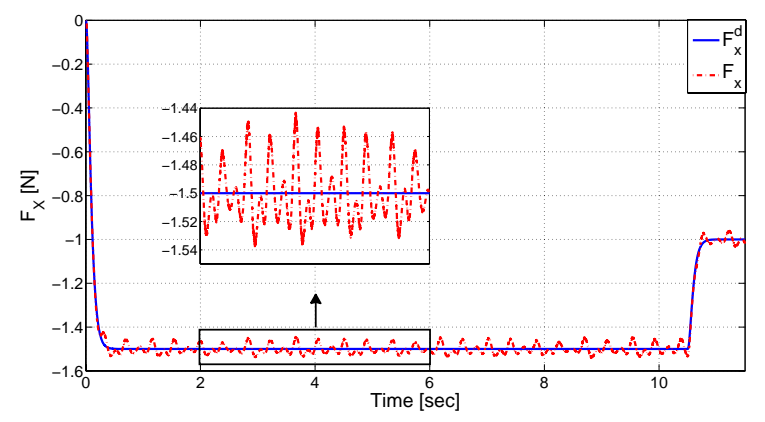

Fig. 6. Evolution of the contact force and its variable reference along $X$ axis in the nominal case

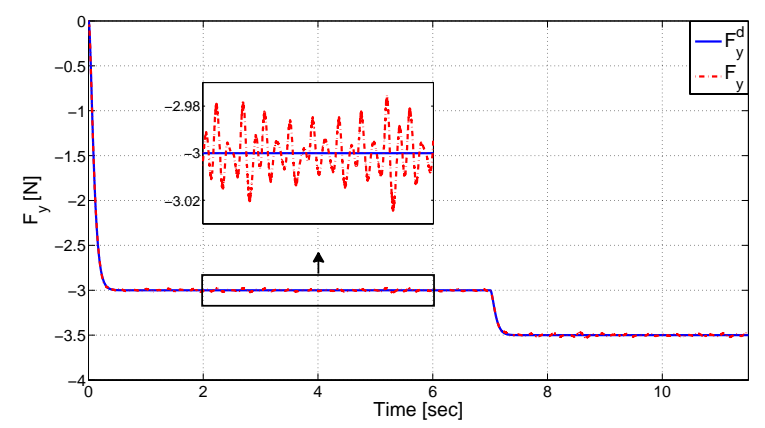

Fig. 7. Evolution of the contact force and its variable reference in the nominal case along $Y$ axis

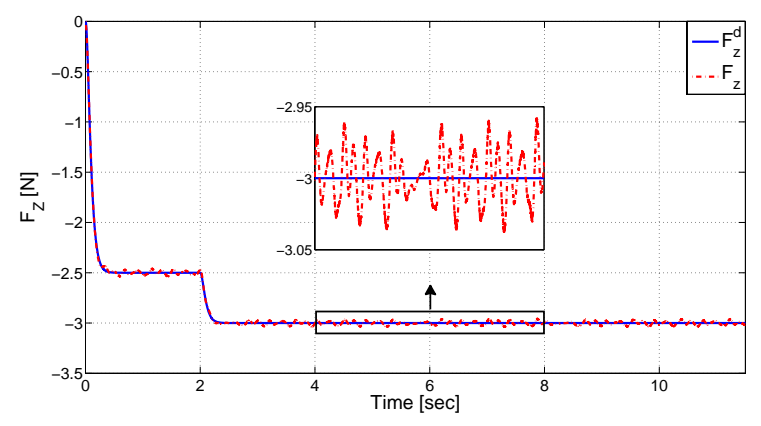

Fig. 8. Evolution of the contact force and its variable reference in the nominal case along $Z$ axis 


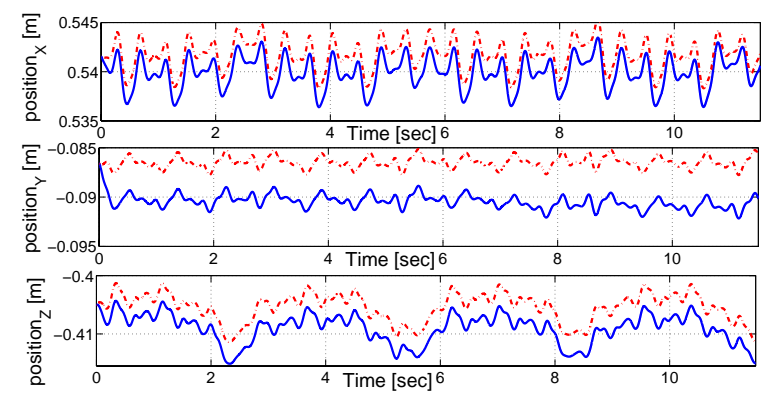

Fig. 9. Compensation of the heart motion along $X, Y$, and $Z$ axis (solid line : end-effector position, dashed line : heart position), in the nominal case

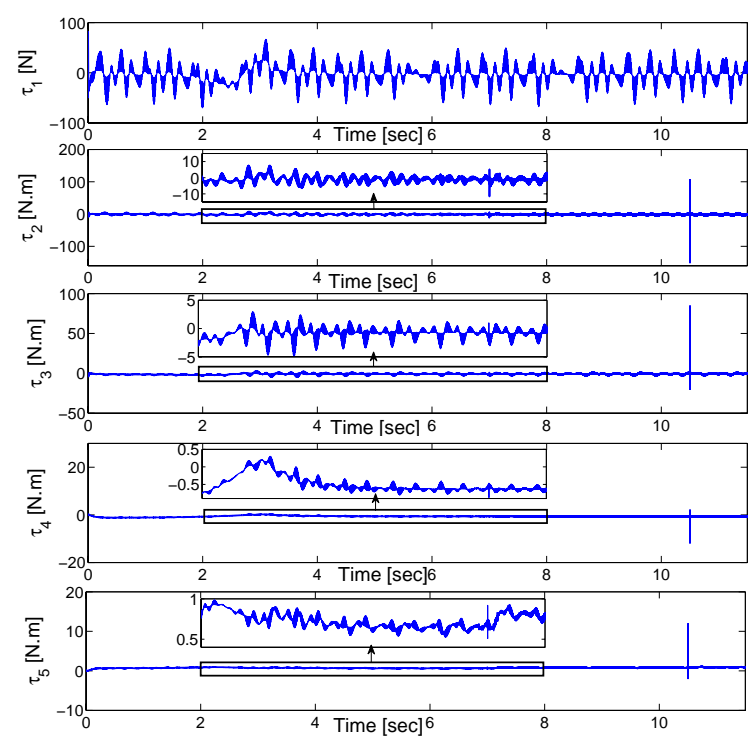

Fig. 10. Evolution of the torques generated by the D2M2 robot in the nominal case

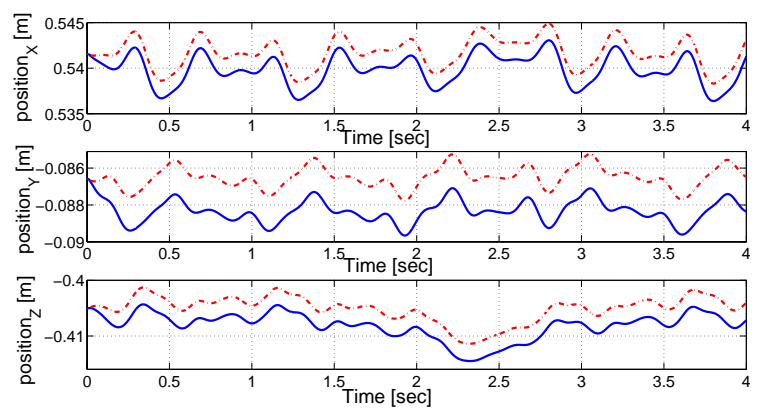

Fig. 11. Compensation of the heart motion along $X, Y$, and $Z$ axis (solid line : end-effector position, dashed line : heart position), in the case of parameters uncertainty consideration

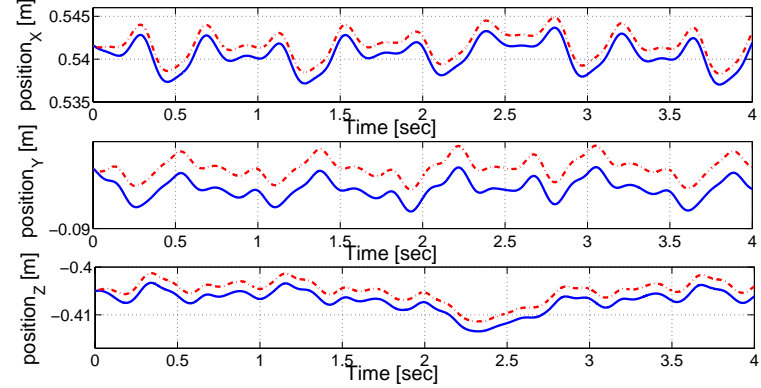

Fig. 12. Compensation of the heart motion along $X, Y$, and $Z$ axis (solid line : end-effector position, dashed line : heart position), in the case of stiffness uncertainty consideration
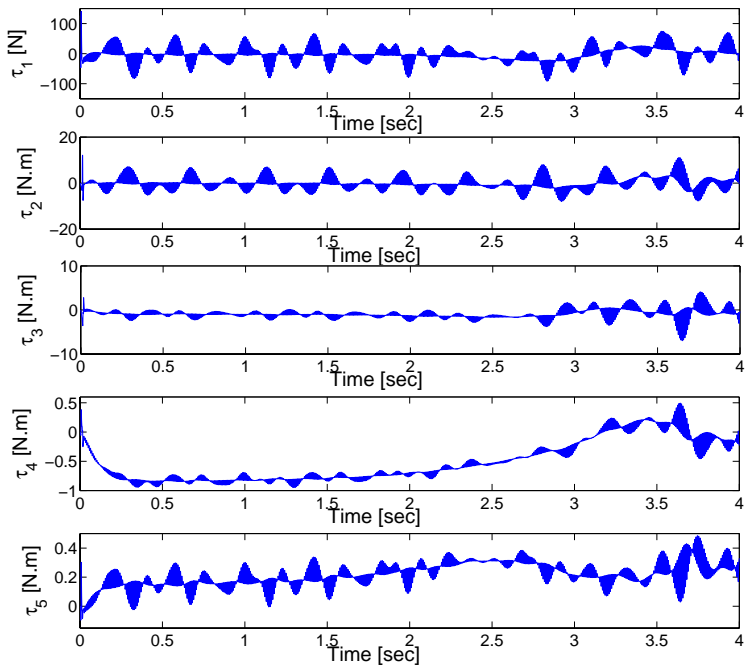

Fig. 13. Evolution of the generated torques versus time in the case of stiffness uncertainty consideration 